\title{
Pim-1 kinase as cancer drug target: An update (Review)
}

\author{
YERNAR TURSYNBAY ${ }^{1 *}$, JINFU ZHANG $^{2}, \mathrm{ZHI} \mathrm{LI}^{3}$, TURSONJAN TOKAY ${ }^{4}$, \\ ZHAXYBAY ZHUMADILOV ${ }^{4}$, DENGLONG WU ${ }^{5}$ and YINGQIU XIE ${ }^{1^{*}}$
}

\begin{abstract}
${ }^{1}$ Department of Biology, Nazarbayev University School of Science and Technology, Astana 010000, Republic of Kazakhstan; ${ }^{2}$ Institute of International Medical Research, Department of Urology and Andrology, Tongren Hospital, Shanghai Jiao Tong University School of Medicine, Shanghai 200336; ${ }^{3}$ Department of Pathology, Sun Yat-sen University, Guangzhou 510080, P.R. China; ${ }^{4}$ Center for Life Sciences, National Laboratory Astana, Nazarbayev University, Astana 010000, Republic of Kazakhstan; ${ }^{5}$ Department of Urology, Tong Ji Hospital, Tong Ji University, Shanghai 200065, P.R. China
\end{abstract}

Received October 22, 2015; Accepted December 8, 2015

DOI: $10.3892 /$ br.2015.561

\begin{abstract}
Proviral integration site for Moloney murine leukemia virus-1 (Pim-1) is a serine/threonine kinase that regulates multiple cellular functions such as cell cycle, cell survival, drug resistance. Aberrant elevation of Pim-1 kinase is associated with numerous types of cancer. Two distinct isoforms of Pim-1 (Pim-1S and Pim-1L) show distinct cellular functions. Pim-1S predominately localizes to the nucleus and Pim-1L localizes to plasma membrane for drug resistance. Recent studies show that mitochondrial Pim-1 maintains mitochondrial integrity. Pim-1 is emerging as a cancer drug target, particularly in prostate cancer. Recently the potent new functions of Pim-1 in immunotherapy, senescence bypass, metastasis and epigenetic dynamics have been found. The aim of the present updated review is to provide brief information regarding networks of Pim-1 kinase and focus on its recent advances as a novel drug target.
\end{abstract}

\section{Contents}

1. Introduction

2. Pim-1 as an immunotherapy target

3. Pim-1 as a drug resistance target

4. Pim-1 as a senescence regulator

5. Pim-1 as a prostate cancer biomarker

6. Pim-1 as an epigenetic dynamics regulator

7. Pim-1 as a metastatic target

8. Pim-1 as a crosstalk signaling pathway target

9. Conclusion

Correspondence to: Professor Yingqiu Xie, Department of Biology, Nazarbayev University School of Science and Technology, Astana 010000, Republic of Kazakhstan

E-mail: xieautumnus@yahoo.com

*Contributed equally

Key words: Pim-1, mitochondrial Pim1, drug target, update

\section{Introduction}

Proviral integration site for Moloney murine leukemia virus-1 (Pim-1) kinase is observed to interact with numerous proteins participating in various signaling pathways (Fig. 1) $(1,2)$. The Pim-1 gene was originally identified as a proviral integration site for Moloney murine leukemia virus-1. Pim-1 is a proto-oncogene that encodes a serine/threonine kinase, which has a crucial role in oncogenesis (3). This proto-oncogene was originally found in hematopoietic cells as a member of the Pim family (Pim-1, Pim-2 and Pim-3). Transcription of Pim-1 can be activated by several interleukins, such as interleukin-2 (IL-2), IL-3 and IL-6. It has been shown that the Pim-1 kinase has an essential role in cytokine-induced signal transduction by controlling transcription factors (4). Upregulation of Pim-1 is correlated with cell proliferation induced by mitogens or cytokines, while downregulation of Pim-1 is correlated with growth retention due to the absence of cytokines (3). Additionally, deficiency of Pim-1 kinase leads to failure in cell survival and growth (1-3). Recent studies have shown that Pim-1 is required in drug resistance and has important roles in prostate cancer. In addition, new functions of Pim-1 have been revealed in immunotherapy, senescence bypass, epigenetic dynamics and cancer metastasis.

Pim-1 has two isoforms, Pim-1L and Pim-1S, which are encoded by the Pim-1 gene due to different translation initiation sites. Human Pim-1L is the longer isoform with a molecular mass of $44 \mathrm{kDa}$, while Pim-1S is the shorter isoform with a molecular mass of $33 \mathrm{kDa}$. Compared to Pim-1S, Pim-1L contains an additional proline-rich PXXP motif at the N-terminus. The extra domain of Pim-1L may allow Pim-1L to interact with more proteins and crosstalk with more signaling networks. For example, our previous study identified that the proline-rich domain of Pim-1L directly interacts with the SH3 domain of Etk (5). Notably, Pim-1S predominately localizes into the nucleus and cytosol; however, Pim-1L mainly localizes at the plasma membrane in prostate cancer cells (5). This cellular localization difference indicates the distinct role of Pim-1L and Pim-1S in cancer. Of note, human Pim-1 kinase protein sequences are similar to that of murine pim-1 kinase (6). In addition, human Pim-1S 
and Pim-1L have a hinge region and a catalytic domain (7). The Pim-1 kinase can be inactivated through altering lysine to methionine in the kinase domain (at position 67 of Pim-1S) (8).

Pim-1 transcription can be activated by interleukins followed by signaling transduction to the nucleus through two families of proteins, Janus kinase (JAK)/signal transducers and activators of transcription (STAT) (Fig. 1). STAT proteins can increase the expression of Pim-1 kinase by binding to the promoter of the Pim-1 gene. This is widely found in the classical upstream of the Pim-1 signaling pathway. However, a previous study indicated that Pim-1 can, in turn, downregulate the JAK/STAT pathway (9). In detail, Pim-1 expression is induced by STAT3 and STAT5 whereupon Pim-1 kinase phosphorylates and stabilizes SOCS1 and SOCS3. Upon phosphorylation, SOCS proteins become more suppressive by interacting with active JAK proteins and blocking phosphorylation of STAT proteins (Fig. 1) (10). In addition, phosphorylated STAT3 triggers Pim-1 expression during human pulmonary hypertension, which in turn contributes to proliferation of smooth muscle cells (11). Thus, Pim-1 may form a feedback loop with the JAK/STAT pathway for tight regulation of its own expression and function. Numerous Pim-1 phosphorylation substrates have been identified, which are involved in cell cycle, cell growth and cell survival (Fig. 1). For example, Pim-1 phosphorylates cell cycle regulator $\mathrm{p} 21$, which thereby dissociates $\mathrm{p} 21$ with proliferating cell nuclear antigen binding to regulate cell cycle and proliferation (12) and phosphorylates p27 to promote cell cycle progression (13). Cell survival depends on signals that inhibit apoptosis. One of the main regulators of cell survival is the B-cell lymphoma-2 (Bcl-2) family comprising Bcl-2, Bcl-xL and myeloid cell leukemia-1 (MCL-1) (14). According to studies by Kumar et al (15), the inactivation of Bcl-2-associated death promoter (BAD) can occur due to phosphorylation at Ser-75 by Pim-1, thus strengthening the dissociation of Bak with Bcl-xL. Therefore, Pim-1 is important for Bcl-xL pro-survival effect. This statement was further supported by the fact that inhibition of Pim-1 kinase suppresses phosphorylation of Bad, which in turn increases LY294002-induced apoptosis in prostate cancer LNCaP cells (15). In addition, multiple signaling networks are regulated by Pim-1 and have been reviewed by numerous researchers suggesting that Pim-1 may be a master regulator of cell function (1) (Fig. 1). The following text will focus on the essential new functions of Pim-1 in cancer.

\section{Pim-1 as an immunotherapy target}

Increasing evidence has shown that Pim-1 would be a novel and essential drug target in numerous types of cancer (16), in particular prostate cancer (17). Pim-1 transcription is regulated by interleukins (ILs), which implicates that Pim-1 can be a potential target for immunotherapy. Our previous study showed that IL-6 can induce Pim-1L and Pim-1S expression (5). Treatment with neutralized IL-6 antibody, results in the decrease of Pim-1L and Pim-1S expression in prostate cancer cells (5). This suggests that targeting Pim-1 has a great potential in immunotherapy.

Directly targeting Pim-1 using a specific monoclonal antibody to Pim-1 has been tested in preclinical studies.
Treatment with the specific antibody to Pim-1 (mAb P9) in SCID mice inoculated with DU145 cells subcutaneously decreased the tumor growth. Additionally, the growth rate of tumors generated from C57BL/6 mice inoculated with TRAMP-C1 cells also was decreased by this antibody (18). Antibody P9 induces apoptotic pathway by specific interaction with Pim-1. They also found that the treatment with Pim-1 antibody P9 significantly inhibited the level of Pim-1 kinase in prostate cancer cell lines, such as PC-3, DU145 and TRAMP-C1 with changes in protein kinase B (or AKT), heat-shock protein 90 and caspase pathways (18). A previous study also showed that the treatment with P9 decreased the growth of human leukemia cell lines (19). Our previous studies identified that Pim-1L (44 kDa) expresses on cell membrane to mediate drug resistance in prostate cancer cells $(5,20)$. Combined treatment with the Pim-1 antibody P9 and chemotherapy drugs decreased prostate cancer cell growth (18).

Human cluster of differentiation 4 [CD4(+)] CD25(high) FOXP3(+) T regulatory cells ( $\mathrm{T}$ regs), which have functional plasticity, can differentiate into effector $\mathrm{T}$ cells induced by inflammation (21). FOXP3 is a specific transcription factor that determines development of $\mathrm{T}$ regs and is critical for obtaining the inhibitory abilities of $\mathrm{T}$ regs (22). According to recent studies FOXP3 is regulated through phosphorylation, which affects its DNA binding ability and stability $(22,23)$. Pim-1 expression can be regulated through $\mathrm{T}$ cell receptor signaling and IL- 6 in in vitro-expanded $\mathrm{T}$ regs (21). Recent studies have shown that human FOXP3 is phosphorylated by Pim-1 kinase at Ser-422, which blocks FOXP3 chromatin-binding activity for expression of target genes. When in vitro-expanded $\mathrm{T}$ reg cells were treated with a Pim-1-specific inhibitor, 3-cyano-4-phenyl-6(3-bromo-6-hydroxy)phenyl-2(1H)-pyridone, they exhibited increased suppressive activity. This means that Pim-1 kinase diminishes the suppressive activity of in vitro-expanded $\mathrm{T}$ regs by inhibiting human FOXP3 (21). From several studies, it has been revealed that $\mathrm{T}$ regs may be one of the reasons for unsuccessful cancer immunotherapies due to inhibiting tumor elimination and activity of tumor-associated antigen-specific lymphocytes (24). Therefore, the efficiency of antitumor immunotherapies can be improved through suppression of $\mathrm{T}$ regs via inhibition of FOXP3 by targeting Pim-1 kinase.

\section{Pim-1 as a drug resistance target}

Emerging evidence has shown that Pim-1 kinase has been associated with the drug-resistant abilities of cancer cells (25). Pim-1 mediates drug resistance through interaction with and phosphorylation of Etk (5), P-glycoprotein (Pgp) (26), breast cancer resistant protein (BCRP) (20) and fms-like tyrosine kinase 3 (FLT3) $(27,28)$ (Fig. 2). The original findings on Pim-1-mediated drug resistance come from the early study that Pim-1 overexpression allows cells to undergo prolonged survival upon withdrawal of IL-3 (29). Following this, Pim-1-mediated drug resistance in prostate cancer was identified as a mechanism of inhibiting p53-induced apoptosis (5). Mechanistically, Pim-1L competes with p53 to bind non-receptor tyrosine kinase Etk. Etk signaling has an important role in this drug 


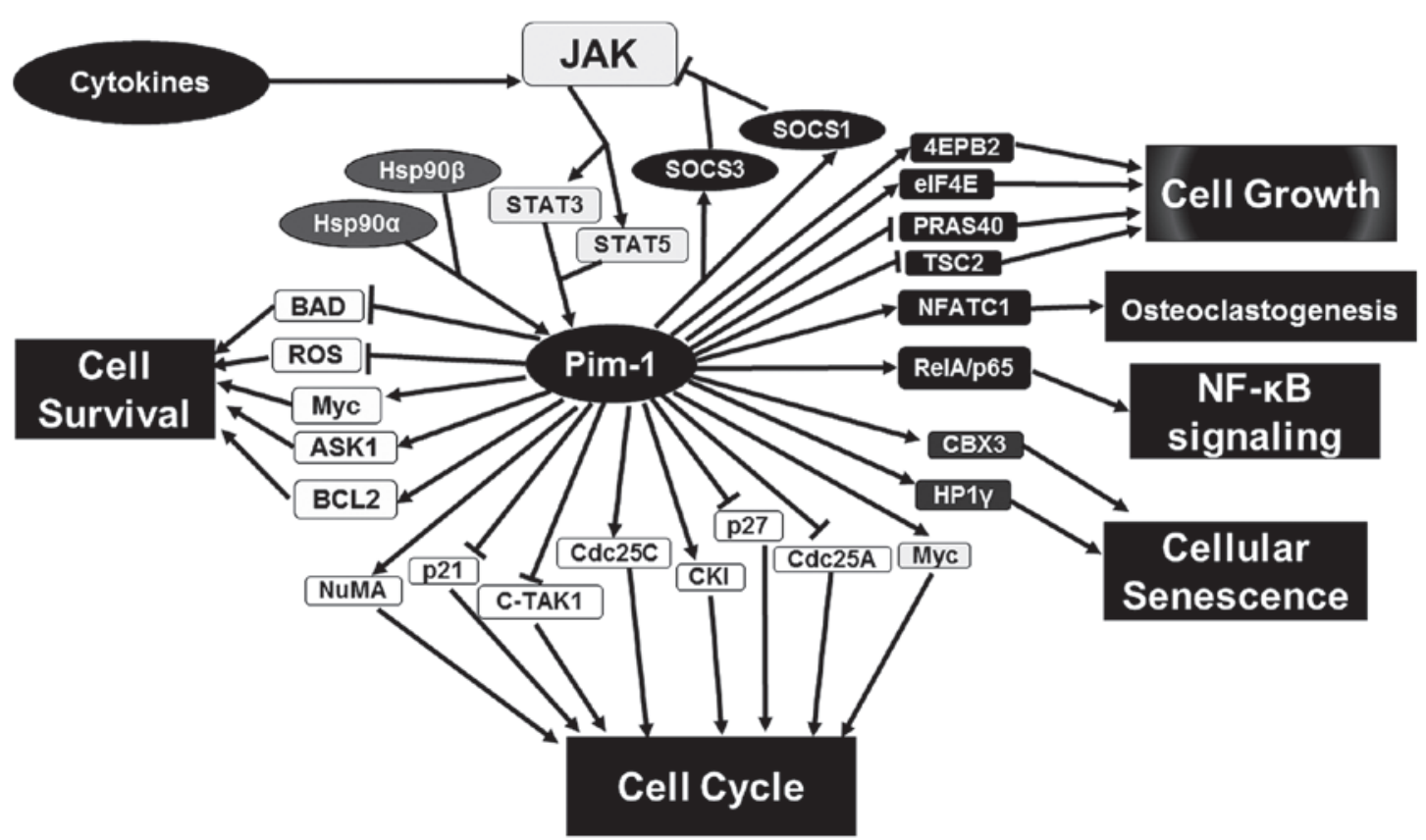

Figure 1. Proviral integration site for Moloney murine leukemia virus-1 (Pim-1) is regulated through the Janus kinase (JAK)/signal transducers and activators of transcription (STAT) pathway and regulates multiple signaling pathways, such as cell survival, cell cycle and cell growth.

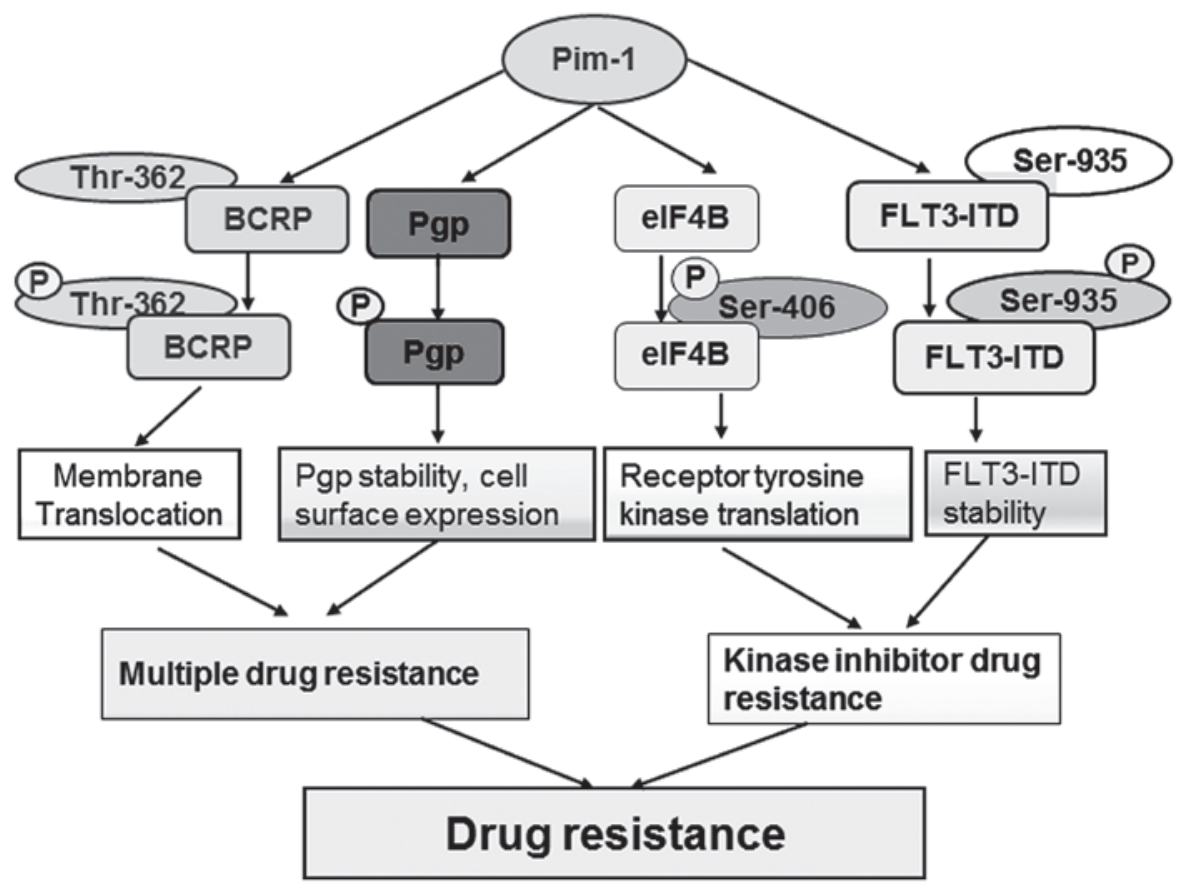

Figure 2. Proviral integration site for Moloney murine leukemia virus-1 (Pim-1) promotes cancer drug resistance. Phosphorylation of breast cancer resistant protein (BCRP), P-glycoprotein (Pgp) and fms-like tyrosine kinase 3 (FLT3)-internal tandem duplication (ITD) leads to changes in their stability or cellular localization, which results in decreased efficiency of antitumor therapies. Thus, Pim-1 is a promising antitumor target.

resistance as Pim-1L, but not Pim-1S, directly interacts with Etk at the plasma membrane while Etk signaling can promote cell survival by inhibiting p53 (30). Thus, Pim-1L showed a higher ability to protect the prostate cancer cells to undergo apoptosis induced by chemotherapy drugs. At that time, it was unclear whether Pim-1 mediated drug resistance was only due to reduced cell apoptosis or through a mechanism of multiple drug resistance mediated by adenosine triphosphate-binding cassette (ABC) drug transporters. Subsequent discoveries benefited from a yeast two-hybrid system using full-length Pim-1L to screen novel Pim-1L-binding proteins. Our first study regarding Pim-1L-mediated multiple drug resistance explained the molecular mechanism that Pim-1L phosphorylates BCRP at Thr-362 resulting in BCRP dimerization and its translocation to the plasma membrane (20). This suggests that translocation of the phosphorylated $\mathrm{ABC}$ transporter by Pim-1 promotes drug resistance via efflux drugs outside of the cells $(10,20)$. Pgp is another member of the ABC family (31). 
It is known that Pgp must translocate to the plasma membrane to enhance drug efflux activity (32). Pim-1 kinase also phosphorylates Pgp and protects it from proteasomal degradation through stabilizing Pgp and enhancing its cell surface expression (26). Combined inhibitors of Pgp and Pim-1 enhance drug efficiency by increased apoptosis of drug resistant cancer cells (26).

In addition to Etk, another tyrosine kinase that interacts with Pim-1 for drug resistance is FLT3. FLT3 is a receptor tyrosine kinase that can be found in normal cells, as well as in cancerous cells (33). However, FLT3 tends to mutate by internal tandem duplication (ITD), which accounts for $30 \%$ of acute myeloid leukemia (AML). AML patients with FLT3-ITD frequently develop resistance to FLT3 inhibitors. It has been shown that STAT5 can be activated by mislocalized and phosphorylated FLT3-ITD, which in turn promotes expression of Pim-1 kinase (34-36). Recently, our previous study revealed that Pim-1 participates in a positive-feedback loop regulating FLT3-ITD expression and stability through phosphorylation at Ser-935. Therefore, Pim-1 kinase facilitates abnormal signaling of FLT3-ITD in cancer cells, and enhancing their drug resistance (37). Combined inhibition of Pim-1 and FLT3 increases cancer cell sensitivity to either drug alone (37).

\section{Pim-1 as a senescence regulator}

Cellular senescence can be described as an arrest of the proliferative abilities of the cell, so that the cell loses the ability to divide (38). Certain characteristics of senescent cells are heterochromatin formation and telomere shortening. It has long been believed that cellular senescence serves as a protective mechanism against cancer (39). The exact role of oncogene-induced senescence in cancer is largely unknown. Recently, it was revealed that Pim-1 expression is elevated upon aging in human fibroblast cells and Pim-1 inhibition reduces replicative and oncogene-induced senescence (40). In addition, it has been shown that expression of Pim-1 is activated through IL-6/STAT3 signaling, thus Pim-1 mediates cytokine-induced cellular senescence (40). Notably, it was found that Pim-1 kinase has the potential to rejuvenate human cardiac progenitor cells (hCPCs). According to obtained results, hCPC cell lines transduced with lentivirus for overexpressing Pim-1 were less susceptible to replicative senescence, had longer telomeres and increased abilities to proliferate (41). These two contradictive findings provide a foundation to further study in this area. Results that state participation of Pim-1 in premature aging via heterochromatin formation make the kinase a potential target in activating cellular senescence through cytokines in cancer therapy. By contrast, the observations showing that Pim-1 kinase has the capacity to rejuvenate hCPC also makes Pim-1 a potential target in deactivating the rejuvenation process in tumor cells. Furthermore, recent new findings show that nuclear localized Pim-1 (refer to Pim-1S) can promote senescence bypass of hCPC stem cells through downregulation of p16 and p53 (42). The new finding that mitochondrial localization of Pim-1 (mito-Pim1) increases hCPC cell survival and decreases apoptosis further supports that distinct cellular localization of Pim-1 fine-tunes the signaling networks for differential functions, such as maintaining mitochondrial integrity, energy and survival for senescence bypass. However, which factors determine the functional switch of Pim-1 in differential genetic context should be further investigated.

\section{Pim-1 as a prostate cancer biomarker}

Elevation of Pim-1 kinase has been found in numerous types of cancer, in particular male hormone-related prostate cancer (2). Tissue microarray analysis using Pim-1S (17) and Pim-1L antibodies (5) showed that Pim-1S and Pim-1L are largely upregulated only in the advanced, but not in the early, stage of prostate cancer. Thus, Pim-1S and Pim-1L can be used as a biomarker for prostate cancer. However, these two isoforms of Pim-1 show distinct roles in hormone-regulated signaling. Androgen receptor (AR) has a central role in prostate cancer progression. Pim-1S and Pim-1L phosphorylate AR at different sites. Pim-1S and Pim-1L can interact with and phosphorylate AR at Ser-213, but only Pim-1L can phosphorylate AR at Thr-850 (43). Pim-1S and Pim-1L mediated phosphorylation results in recruiting the distinct ubiquitin E3 ligase. Our previous study showed that Pim-1S-induced Ser-213 phosphorylation of AR promotes AR degradation through ubiquitin E3 ligase Mdm2 depending on cell cycle (43). However, Pim-1L-induced Thr-850 phosphorylation stabilizes AR through ubiquitin E3 ligase RNF6 and enhances AR target gene transcription under low-androgen conditions (43). More data showed that Pim-1S and Pim-1L can promote prostate cancer cell growth even in low-androgen conditions (43). These data suggest that Pim-1 has pivotal roles in hormone refractory prostate cancer. Similar findings were reported that Pim-1S phosphorylates AR at Ser-213 and inhibits AR target genes, such as tumor suppressor genes NKX3.1 $(44,45)$. However, Pim-1S-mediated phosphorylation at Ser-213 also inhibits AR target gene PSA. This paradox between oncogenic Pim-1S and PSA most likely is caused by ubiquitination and degradation of AR following phosphorylation at Ser-213. Pim-1L may switch AR target genes by RNF6, as RNF6 regulates AR target genes specificity (46).

\section{Pim-1 as an epigenetic dynamics regulator}

Phosphorylation of heterochromatin protein $1 \gamma$ at Ser-93 by Pim-1 promotes its binding with histone H3K9me3, which leads to heterochromatin formation and suppression of gene transcriptions responsible for proliferation (40). Another epigenetic regulation of Pim-1 involves oncogenic transcription factor c-Myc (Myc). One study showed that Pim-1 can directly regulate Myc transcriptional activity (47). Pim-1 overexpression alone is not enough to transform benign prostate RWPE1 cell line to malignantly form (48). However, Pim-1 overexpression combined with Myc leads to development of the advanced form of prostate carcinoma (49). Recent studies have shown that Pim-1 associates with Myc and can thereby regulate the epigenetic dynamics of oncogene expression. Pim-1-mediated co-regulation consists of $\sim 20 \%$ of the Myc-regulated genes. Pim-1 phosphorylates histone $\mathrm{H} 3$ at Ser-10 (H3S10) on the nucleosome at the MYC-binding sites. This suggests that Pim-1 regulates transcriptional activation, which contributes to Myc-transforming activity (50). Thus, 
Pim-1 is a transcriptional cofactor of Myc that phosphorylates the chromatin at Myc-binding sites and regulates epigenetic dynamics for cellular transformation.

\section{Pim-1 as a metastatic target}

Given the elevation of Pim-1 in highly advanced stages of cancers, in addition to the important role of Pim-1 in cell survival and proliferation, whether Pim-1 directly regulates cancer cell invasion to induce metastasis remains to be elucidated. Recently, one study showed Pim-1 overexpressing prostate cancer PC3 cells induced tumor invasion to prostate-draining lymph nodes, but also into the lungs to form metastases in a Xenograft model (51). Mechanistically, Pim-1 phosphorylates CXCR4 at Ser-339 for cell migration and invasion (51). The epithelial-mesenchymal transition (EMT) is known to be one of the mechanisms of metastasis. Pim-1 is expressed at high levels in the stroma of human prostate cancer samples (52). Inducible overexpression of Pim-1 in immortalized prostate fibroblast cell lines increased the differentiation of myofibroblasts and transition of cancer-associated fibroblasts (52). Pim-1 in fibroblasts upregulated the expression levels of secreted proteins of extracellular matrix collagen 1A1, chemokine CCL5, and the platelet-derived growth factor receptors (52). In addition, Pim-1 upregulated c-MET, a well-known EMT inducer through translational regulation (53). Pim-1 regulated MET through the control of the translation of c-MET by regulating the phosphorylation of eukaryotic initiation factor 4B (eIF4B) at Ser-406 (53). As c-MET kinase is an inducer of cancer metastasis, these findings suggest Pim-1 may have a significant potential in cancer metastasis by crosstalk with multiple signaling.

\section{Pim-1 as a crosstalk signaling pathway target}

Targeting Pim-1 in prostate cancer would be promising for preventing the cancer recurrence caused by kinase inhibitor drugs in clinical treatment. For example, the PI3K/AKT pathway is a strong signaling pathway that promotes cell proliferation and survival in numerous types of cancer including prostate cancer. However, a single treatment targeting this pathway has been a significant obstacle for therapy efficiency. One of the mechanisms is that Akt inhibition can induce upregulation of numerous receptor tyrosine kinases, such as c-MET, HER2 and insulin receptor growth factor in prostate cancer cells through Pim-1 mediated regulation of translation in a cap-independent manner, but internal ribosome entry-dependent manner (53). Furthermore, Pim-1 inhibition by inhibitor SMI-4a represses the resistance to Akt inhibitor drugs (54).

Numerous Pim-1 inhibitors, such as flavonoid inhibitors (55), isoxazolo[3,4-b]quinoline-3,4(1H,9H)-dione (56), ETP-45299 (57), SGI-1776 (58) and AZD1208 (59), have been developed. They can be classified as the first generation inhibitor (SGI-1776) and the next generation inhibitor (AZD1208) (60). SGI-1776 as the first generation inhibitor is able to inhibit Pim-1S, Pim-1L, Pim-2 and Pim-3 kinases and has high antitumor activity in vivo as well as in vitro (61). The antitumor effect of SGI-1776 could bypass Pim-1 as the inhibitor also suppresses the activity of FLT3 (61). Additionally, it has been shown that SGI-1776 can inhibit cyclin D1, MCL and Myc (61).
SGI-1776 could inhibit Pgp-mediated efflux of drugs through inhibiting Pim-1 kinase, thus reversing the drug-resistant abilities of tumor cells (27). In addition, this inhibitor decreased the surface expression of Pgp and BCRP in K562/ABCG2 and K562/Pgp cell lines and enhanced apoptosis of Pgp and BCRP overexpressing cells (27). One of the new generation inhibitors is TP-3654, which can also inhibit all isoforms of Pim kinase (Pim-1, Pim-2 and Pim-3), but with a small effect on FLT3 and hERG (62). This is significant as phase I clinical trials of SGI-1776 revealed its cardiotoxicity due to suppression of the cardiac potassium channel (61). The next promising second generation Pim kinase inhibitor is AZD1208, which can inhibit Pim-1S and Pim-1L (59). AZD1208 was found to inhibit phosphorylation of BAD at Ser-112, which is intermediary of pro-survival activity of Pim-1 kinase (63). According to microarray and RNA-sequencing studies, it has been shown that treatment with AZD1208 causes inhibition of the Myc pathway in vitro with acutely treated cancer cells and in vivo with tumor cells that were treated chronically (64). Additionally, it has been shown that AZD1208 inhibits the tumor suppressor signaling pathway mediated by $\mathrm{p} 53$, which is stabilized by overexpression of Pim-1 kinase (65). The fact that AZD1208 can suppress the p53 pathway and that Pim-1 kinase can be activated by hypoxia and radiation to enhance survival of cancer cells suggests the benefit of the combination of radiation therapy and AZD1208 treatment, as prostate cancer cells are more resistant to radiation upon hypoxic response (66). Recent studies have shown in Myc-mediated castration-resistant cancerous cells, AZD1208 increases radiation efficiency during prostate cancer treatment (59). Therefore, AZD1208 as a promising Pim-1 inhibitor can be used for combinational therapy in advanced prostate cancer. Furthermore, the selective inhibitor of the Pim family, DHPCC-9, decreases the metastatic ability of prostate tumors to the lung (51). Recently it has been shown that CD25, a stem cell marker and prognostic marker of survival or relapse in AML, can also be used as a prognostic marker for Pim-1 inhibitor drug response (67). This data implicates the potential of the Pim-1 inhibitor in targeting cancer stem cells, linking the mechanisms and functions of Pim-1 in drug resistance and cancer stem cells. However, whether Pim-1 inhibitors can directly target cancer stem cells requires further investigation. In conclusion, increasing evidence has shown that Pim-1 is emerging as a diagnostic marker and drug target in numerous types of cancer (68).

\section{Conclusion}

Pim-1 kinase is a critical enzyme that is involved in cell growth, differentiation, survival, apoptosis, senescence and drug resistance. Interaction of Pim-1 with different proteins and association with various signaling pathways make it one of the important antitumor targets. Numerous Pim-1 inhibitors are under preclinical studies or clinical trials, such as P9 monoclonal antibodies and AZD1208. An increasing number of new Pim-1 inhibitors are still developing and undergoing preclinical investigations. These efforts further suggest that Pim-1 is believed to be a master drug target in numerous types of cancer. In addition, the fact that Pim-1 kinase inhibits transcriptional activity of FOXP3 makes it an even more noteworthy antitumor target, as it is thought that $\mathrm{T}$ regs are responsible for decreasing the efficiency of cancer immunotherapies. Furthermore, Pim-1 
can promote drug resistance, a trait of cancer stem cells, through interaction with and phosphorylation of Pgp, BCRP and FLT3-ITD, which links Pim-1 as a promising targeted therapy in cancer stem cells (69). The new findings of the role of Pim-1 in cellular senescence in differential cancer microenvironment (70) allow us to be cautious for cancer treatment in individual therapy. As Pim-1 is a potential biomarker of prostate cancer and crosstalk with numerous signaling pathways, targeting Pim-1 in immunotherapy and personalized therapy would be of great significance for the next generation of precision medicine in cancer.

\section{Acknowledgements}

The study was supported in part by the seed grant to Y.X.

\section{References}

1. Narlik-Grassow M, Blanco-Aparicio C and Carnero A: The PIM family of serine/threonine kinases in cancer. Med Res Rev 34: 136-159, 2014

2. Warfel NA and Kraft AS: PIM kinase (and Akt) biology and signaling in tumors. Pharmacol Ther 151: 41-49, 2015.

3. Li J, Loveland BE and Xing PX: Anti-Pim-1 mAb inhibits activation and proliferation of T lymphocytes and prolongs mouse skin allograft survival. Cell Immunol 272: 87-93, 2011.

4. Aho TLT, Sandholm J, Peltola KJ, Mankonen HP, Lilly M and Koskinen PJ: Pim-1 kinase promotes inactivation of the pro-apoptotic Bad protein by phosphorylating it on the Ser112 gatekeeper site. FEBS Lett 571: 43-49, 2004.

5. Xie Y, Xu K, Dai B, Guo Z, Jiang T, Chen H and Qiu Y: The $44 \mathrm{kDa}$ Pim-1 kinase directly interacts with tyrosine kinase Etk/BMX and protects human prostate cancer cells from apoptosis induced by chemotherapeutic drugs. Oncogene 25: 70-78, 2006

6. Saris CJM, Domen J and Berns A: The pim-1 oncogene encodes two related protein-serine/threonine kinases by alternative initiation at AUG and CUG. EMBO J 10: 655-664, 1991.

7. Kumar A, Mandiyan V, Suzuki Y, Zhang C, Rice J, Tsai J, Artis DR, Ibrahim P and Bremer R: Crystal structures of proto-oncogene kinase Pim1: A target of aberrant somatic hypermutations in diffuse large cell lymphoma. J Mol Biol 348: 183-193, 2005.

8. Bachmann M and Möröy T: The serine/threonine kinase Pim-1. Int J Biochem Cell Biol 37: 726-730, 2005.

9. Yin J, Shine L, Raycroft F, Deeti S, Reynolds A, Ackerman KM, Glaviano A, O'Farrell S, O'Leary O, Kilty C, et al: Inhibition of the Pim1 oncogene results in diminished visual function. PLoS One 7: e52177, 2012.

10. Magnuson NS, Wang Z, Ding G and Reeves R: Why target PIM1 for cancer diagnosis and treatment? Future Oncol 6: 1461-1478, 2010.

11. Hofmann AD, Takahashi T, Duess J, Gosemann JH and Puri P: Increased expression of activated pSTAT3 and PIM-1 in the pulmonary vasculature of experimental congenital diaphragmatic hernia. J Pediatr Surg 50: 908-911, 2015.

12. Zhang Y, Wang Z and Magnuson NS: Pim-1 kinase-dependent phosphorylation of p21Cip1/WAF1 regulates its stability and cellular localization in H1299 cells. Mol Cancer Res 5: 909-922, 2007.

13. Morishita D, Katayama R, Sekimizu K, Tsuruo T and Fujita N: Pim kinases promote cell cycle progression by phosphorylating and down-regulating p27Kip1 at the transcriptional and posttranscriptional levels. Cancer Res 68: 5076-5085, 2008.

14. Lam LT, Zhang H, Xue J, Hessler P, Tahir SK, Chen J, Jin S, Souers AJ and Leverson JD: Colorectal cancer cell lines with high BCL-XL and low MCL-1 expression are sensitive to a potent and selective BCL-XL inhibitor. Cancer Res 74 (Suppl 19): 2759, 2014.

15. Kumar JK, Ping RYS, Teong HF, Goh S and Clément MV: Activation of a non-genomic Pim-1/Bad-Pser75 module is required for an efficient pro-survival effect of $\mathrm{Bcl}-\mathrm{xL}$ induced by androgen in LNCaP cells. Int J Biochem Cell Biol 43: 594-603, 2011.
16. Block KM, Hanke NT, Maine EA and Baker AF: IL-6 stimulates STAT3 and Pim-1 kinase in pancreatic cancer cell lines. Pancreas 41: 773-781, 2012.

17. Dhanasekaran SM, Barrette TR, Ghosh D, Shah R, Varambally S, Kurachi K, Pienta KJ, Rubin MA and Chinnaiyan AM: Delineation of prognostic biomarkers in prostate cancer. Nature 412: 822-826, 2001

18. Hu XF, Li J, Vandervalk S, Wang Z, Magnuson NS and Xing PX: PIM-1-specific mAb suppresses human and mouse tumor growth by decreasing PIM-1 levels, reducing Akt phosphorylation, and activating apoptosis. J Clin Invest 119: 362-375, 2009.

19. Li J, Hu XF, Loveland BE and Xing PX: Pim-1 expression and monoclonal antibody targeting in human leukemia cell lines. Exp Hematol 37: 1284-1294, 2009.

20. Xie Y, Xu K, Linn DE, Yang X, Guo Z, Shimelis H, Nakanishi T, Ross DD, Chen H, Fazli L, et al: The 44-kDa Pim-1 kinase phosphorylates BCRP/ABCG2 and thereby promotes its multimerization and drug-resistant activity in human prostate cancer cells. J Biol Chem 283: 3349-3356, 2008.

21. Li Z, Lin F, Zhuo C, Deng G, Chen Z, Yin S, Gao Z, Piccioni M, Tsun A, Cai S, et al: PIM1 kinase phosphorylates the human transcription factor FOXP3 at serine 422 to negatively regulate its activity under inflammation. J Biol Chem 289: 26872-26881, 2014.

22. Nie H, Zheng Y, Li R, Guo TB, He D, Fang L, Liu X, Xiao L, Chen X, Wan B, et al: Phosphorylation of FOXP3 controls regulatory $\mathrm{T}$ cell function and is inhibited by TNF- $\alpha$ in rheumatoid arthritis. Nat Med 19: 322-328, 2013.

23. Morawski PA, Mehra P, Chen C, Bhatti T and Wells AD: Foxp3 protein stability is regulated by cyclin-dependent kinase 2 . J Biol Chem 288: 24494-24502, 2013

24. Oleinika K, Nibbs RJ, Graham GJ and Fraser AR: Suppression, subversion and escape: The role of regulatory $\mathrm{T}$ cells in cancer progression. Clin Exp Immunol 171: 36-45, 2013.

25. Isaac M, Siu A and Jongstra J: The oncogenic PIM kinase family regulates drug resistance through multiple mechanisms. Drug Resist Updat 14: 203-211,2011.

26. Xie Y, Burcu M, Linn DE, Qiu Y and Baer MR: Pim-1 kinase protects P-glycoprotein from degradation and enables its glycosylation and cell surface expression. Mol Pharmacol 78: 310-318, 2010.

27. Natarajan K, Bhullar J, Shukla S, Burcu M, Chen ZS Ambudkar SV and Baer MR: The Pim kinase inhibitor SGI-1776 decreases cell surface expression of P-glycoprotein (ABCB1) and breast cancer resistance protein (ABCG2) and drug transport by Pim-1-dependent and -independent mechanisms. Biochem Pharmacol 85: 514-524, 2013.

28. Kim KT, Baird K, Ahn JY, Meltzer P, Lilly M, Levis M and Small D: Pim-1 is up-regulated by constitutively activated FLT3 and plays a role in FLT3-mediated cell survival. Blood 105: 1759-1767, 2005

29. Lilly M, Sandholm J, Cooper JJ, Koskinen PJ and Kraft A: The PIM-1 serine kinase prolongs survival and inhibits apoptosis-related mitochondrial dysfunction in part through a bcl-2-dependent pathway. Oncogene 18: 4022-4031, 1999.

30. Jiang T, Guo Z, Dai B, Kang M, Ann DK, Kung HJ and Qiu Y: Bi-directional regulation between tyrosine kinase Etk/BMX and tumor suppressor p53 in response to DNA damage. J Biol Chem 279: 50181-50189, 2004

31. Riganti C, Gazzano E, Gulino GR, Volante M, Ghigo D and Kopecka J: Two repeated low doses of doxorubicin are more effective than a single high dose against tumors overexpressing P-glycoprotein. Cancer Lett 360: 219-226, 2015.

32. Gribar JJ, Ramachandra M, Hrycyna CA, Dey $S$ and Ambudkar SV: Functional characterization of glycosylation-deficient human P-glycoprotein using a vaccinia virus expression system. J Membr Biol 173: 203-214, 2000.

33. Meshinchi S and Appelbaum FR: Structural and functional alterations of FLT3 in acute myeloid leukemia. Clin Cancer Res 15: 4263-4269, 2009.

34. Schmidt-Arras D, Böhmer SA, Koch S, Müller JP, Blei L, Cornils H, Bauer R, Korasikha S, Thiede C and Böhmer FD: Anchoring of FLT3 in the endoplasmic reticulum alters signaling quality. Blood 113: 3568-3576, 2009.

35. Stout BA, Bates ME, Liu LY, Farrington NN and Bertics PJ: IL-5 and granulocyte-macrophage colony-stimulating factor activate STAT3 and STAT5 and promote Pim-1 and cyclin D3 protein expression in human eosinophils. J Immunol 173: 6409-6417, 2004. 
36. Choudhary C, Olsen JV, Brandts C, Cox J, Reddy PN, Böhmer FD, Gerke V, Schmidt-Arras DE, Berdel WE, Müller-Tidow C, et al: Mislocalized activation of oncogenic RTKs switches downstream signaling outcomes. Mol Cell 36: 326-339, 2009.

37. Natarjan K, Xie Y, Burcu M, Linn DE, Qui Y and Baer MR: Pim-1 kinase phosphorylates and stabilizes $130 \mathrm{kDa}$ FLT3 and promotes aberrant STAT5 signaling in acute myeloid leukemia with FLT3 internal tandem duplication. PLoS One 8: e76453, 2013.

38. Lanigan F, Geraghty JG and Bracken AP: Transcriptional regulation of cellular senescence. Oncogene 30: 2901-2911, 2011

39. Vargas J, Feltes BC, Poloni JG and Bonatto D: Senescence, an endogenous anticancer mechanism. Fronti Biosci (Landmark Ed.) 17: 2616-2643, 2012.

40. Jin B, Wang Y, Wu CL, Liu KY, Chen H and Mao ZB: PIM-1 modulates cellular senescence and links IL-6 signaling to heterochromatin formation. Aging Cell 13: 879-889, 2014.

41. Mohsin S, Khan M, Nguyen J, Alkatib M, Siddiqi S, Hariharan N, Wallach K, Monsanto M, Gude N, Dembitsky W, et al: Rejuvenation of human cardiac progenitor cells with Pim-1 kinase. Circ Res 113: 1169-1179, 2013.

42. Samse K, Emathinger J, Hariharan N, Quijada P, Ilves K, Völkers M, Ormachea L, De La Torre A, Orogo AM, Alvarez R, et al: Functional ffect of Pim1 depends upon intracellular localization in human cardiac progenitor cells. J Biol Chem 290: 13935-13947, 2015

43. Linn DE, Yang X, Xie Y, Alfano A, Deshmukh D, Wang X, Shimelis $\mathrm{H}$, Chen $\mathrm{H}, \mathrm{Li} \mathrm{W}, \mathrm{Xu} \mathrm{K}$, et al: Differential regulation of androgen receptor by PIM-1 kinases via phosphorylation-dependent recruitment of distinct ubiquitin E3 ligases J Biol Chem 287: 22959-22968, 2012.

44. Ha S, Iqbal NJ, Mita P, Ruoff R, Gerald WL, Lepor H, Taneja SS Lee P, Melamed J, Garabedian MJ, et al: Phosphorylation of the androgen receptor by PIM1 in hormone refractory prostate cancer. Oncogene 32: 3992-4000, 2013

45. Song H, Zhang B, Watson MA and Humphrey PA, Lim H and Milbrandt J: Loss of Nkx3.1 leads to the activation of discrete downstream target genes during prostate tumorigenesis. Oncogene 28: 3307-3319, 2009

46. Xu K, Shimelis H, Linn DE, Jiang R, Yang X, Sun F, Guo Z, Chen $\mathrm{H}, \mathrm{Li}$ W, Chen $\mathrm{H}$, et al: Regulation of androgen receptor transcriptional activity and specificity by RNF6-induced ubiquitination. Cancer Cell 15: 270-282, 2009.

47. Kim J, Roh $\mathrm{M}$ and Abdulkadir SA: Pim1 promotes human prostate cancer cell tumorigenicity and c-MYC transcriptional activity. BMC Cancer 10: 248, 2010

48. Kim J, Eltoum IE, Roh M, Wang J and Abdulkadir SA: Interactions between cells with distinct mutations in c-MYC and Pten in prostate cancer. PLoS Genet 5: e1000542, 2009.

49. Wang J, Kim J, Roh M, Franco OE, Hayward SW, Wills ML and Abdulkadir SA: Pim1 kinase synergizes with c-MYC to induce advanced prostate carcinoma. Oncogene 29: 2477-2487, 2010.

50. Zippo A, De Robertis A, Serafini R and Oliviero S PIM1-dependent phosphorylation of histone $\mathrm{H} 3$ at serine 10 is required for MYC-dependent transcriptional activation and oncogenic transformation. Nat Cell Biol 9: 932-944, 2007.

51. Santio NM, Eerola SK, Paatero I, Yli-Kauhaluoma J, Anizon F, Moreau P, Tuomela J, Härkönen P and Koskinen PJ: Pim kinases promote migration and metastatic growth of prostate cancer xenografts. PLoS One 10: e0130340, 2015.

52. Zemskova MY, Song JH, Cen B, Cerda-Infante J, Montecinos VP and Kraft AS: Regulation of prostate stromal fibroblasts by the PIM1 protein kinase. Cell Signal 27: 135-146, 2015.

53. Cen B, Xiong Y, Song JH, Mahajan S, DuPont R, McEachern K, DeAngelo DJ, Cortes JE, Minden MD, Ebens A, et al: The Pim-1 protein kinase is an important regulator of MET receptor tyrosine kinase levels and signaling. Mol Cell Biol 34: 2517-2532, 2014.

54. Cen B, Mahajan S, Wang W and Kraft AS: Elevation of receptor tyrosine kinases by small molecule AKT inhibitors in prostate cancer is mediated by Pim-1. Cancer Res 73: 3402-3411, 2013.
55. Tong Y, Stewart KD, Thomas S, Przytulinska M, Johnson EF, Klinghofer V, Leverson J, McCall O, Soni NB, Luo Y, et al: Isoxazolo[3,4-b]quinoline-3,4(1H,9H)-diones as unique, potent and selective inhibitors for Pim-1 and Pim-2 kinases: Chemistry, biological activities, and molecular modeling. Bioorg Med Chem Lett 18: 5206-5208, 2008.

56. Holder S, Lilly M and Brown ML: Comparative molecular field analysis of flavonoid inhibitors of the PIM-1 kinase. Bioorg Med Chem 15: 6463-6473, 2007.

57. Blanco-Aparicio C, Collazo AM, Oyarzabal J, Leal JF, Albarán MI, Lima FR, Pequeño B, Ajenjo N, Becerra M, Alfonso P, et al: Pim 1 kinase inhibitor ETP-45299 suppresses cellular proliferation and synergizes with PI3K inhibition. Cancer Lett 300: 145-153, 2011.

58. Yang Q, Chen LS, Neelapu SS and Gandhi V: Combination of Pim kinase inhibitor SGI-1776 and bendamustine in B-cell lymphoma. Clin Lymphoma Myeloma Leuk 13 (Suppl 2): S355-S362, 2013.

59. Keeton E, McEachern K, Alimzhanov M, Wang S, Cao Y, Bao L, Palakurthi S, Grondine M, Chen Y, Dillman K et al: Efficacy and biomarker modulation by AZD1208, a novel, potent and selective pan-Pim kinase inhibitor, in models of acute myeloid leukemia. Cancer Res 72: 2796, 2012.

60. Mondello P, Cuzzocrea S, Mian M: Pim kinases in hematological malignancies: Where are we now and where are we going? J Hematol Oncol 7: 95, 2014.

61. Hospital MA, Green AS, Lacombe C, Mayeux P, Bouscary D and Tamburini J: The FLT3 and Pim kinases inhibitor SGI-1776 preferentially target FLT3-ITD AML cells. Blood 119: 1791-1792, 2012.

62. Foulks JM, Carpenter KJ, Luo B, Xu Y, Senina A, Nix R, Chan A, Clifford A, Wilkes M, Vollmer D, et al: A small-molecule inhibitor of PIM kinases as a potential treatment for urothelial carcinomas. Neoplasia 16: 403-412, 2014.

63. Keeton EK, McEachern K, Dillman KS, Palakurthi S, Cao Y, Grondine MR, Kaur S, Wang S, Chen Y, Wu A, et al: AZD1208, a potent and selective pan-Pim kinase inhibitor, demonstrates efficacy in preclinical models of acute myeloid leukemia. Blood 123: 905-913, 2014

64. Kirschner AN, Wang J, van der Meer R, Anderson PD, Franco-Coronel OE, Kushner MH, Everett JH, Hameed O, Keeton EK, Ahdesmaki M, et al: PIM kinase inhibitor AZD1208 for treatment of MYC-driven prostate cancer. J Natl Cancer Inst 107: dju407, 2014

65. Hogan C, Hutchison C, Marcar L, Milne D, Saville M, Goodlad J, Kernohan N and Meek D: Elevated levels of oncogenic protein kinase Pim-1 induce the p53 pathway in cultured cells and correlate with increased Mdm2 in mantle cell lymphoma. J Biol Chem 283: 18012-18023, 2008.

66. Turaka A, Buyyounouski MK, Hanlon AL, Horwitz EM, Greenberg RE and Movsas B: Hypoxic prostate/muscle PO2 ratio predicts for outcome in patients with localized prostate cancer: Long-term results. Int J Radiat Oncol Biol Phys 82: e433-e439, 2012.

67. Guo Z, Wang A, Zhang W, Levit M, Gao Q, Barberis C, Tabart M, Zhang J, Hoffmann D, Wiederschain D, et al: PIM inhibitors target CD25-positive AML cells through concomitant suppression of STAT5 activation and degradation of MYC oncogene. Blood 124: 1777-1789, 2014.

68. Liang C and Li YY: Use of regulators and inhibitors of Pim-1, a serine/threonine kinase, for tumour therapy (Review). Mol Med Rep 9: 2051-2060, 2014

69. Xie Y and Bayakhmetov S: PIM1 kinase as a promise of targeted therapy in prostate cancer stem cells (Review). Mol Clin Oncol 4: 13-17, 2016.

70. Xie Y, Lu W, Liu S, Yang Q, Carver BS and Chen Z: The essential role of ARF in prostate cancer microenvironment. BJU Int 116: 41-41, 2015 